\title{
Portable Parallel Programming for the Dynamic Load Balancing of Unstructured Grid Applications
}

\author{
Rupak Biswas \\ MRJ Technology Solutions \\ NASA Ames Research Center \\ Moffett Field, CA 94035, USA \\ rhiswas@nas.nasa.gov
}

\author{
Sajal K. Das, Daniel Harvey \\ Dept of Computer Sciences \\ University of North Texas \\ Denton, TX 76203, USA \\ \{das,harvey\}@cs.unt.edu
}

\author{
Leonid Oliker \\ RIACS \\ NASA Ames Research Center \\ Moffett Field, CA 94035, USA \\ oliker@riacs.edu
}

\begin{abstract}
The ability to dynamically adapt an unstruciured grid (or mesh) is a powerfill tool for solving computational prob. lenis with evolving physical features; however, an efficient parallel implementation is rather difficult. particularly from the vicupoint of portability on various multiprocessor platforms. He address this problem by developin; PLUM. an aunnutic and architeclure-independent framework for adcaptive numierical computations in a messuge-passing environment. Portability is demonstrated by comparing performance on an SP2, an Origin2000, and a T3E, without any code modifications. We also present a general-purpose load balancer that utilizes symmetric broadcast networks ( $S B N$ ) as the underlying communication pattern, with a goal to providing a global view of system loads across processors. Experiments on an SP2 and an Origin2000 demonstrate the portability of our approach which achieves superb load balance at the cost of minimial extra overizead.
\end{abstract}

\section{Introduction}

The sucess of paridlel computing in solving real-life, compuation-intensive problems relies on their etticient mapping and cxecution on commercially available mutiprocessor architectures. Whet the algorithms and data stnictures comesponding to these problems are dynamic in narure (i.e., their computationil workloads grow or shrink at runtime) or are intrinsicadly unstructured, mapping them on (1) Listributed -memory parallel machines with dynimic load hallancing olliers considerable challenges. Dynamic load hallancing aims to halance processor workloads at runtime while ninimizing inter-processor comnunication. With the prolificration ol parallicl computing, dyanamic load baliancing hats heconce cxiremely important in severall applications like sciemilic computing. talsk scheduling. sparse matrix compu- tations, parallel discrete event simulation, and data mining.

The ability to dynamically adapt an unstructured mesh is a powertul tool for efficiently solving computational prob lems with evolving physical features. Standard fixed-mesh numerical methods can be made more cost-effective by locally refining and coarsening the mesh to capture these phenomena of interest. Unfortunately, an efficient parillelization of adaptive methods is rather difficult, primarily due to the load imbal ance created by the dynamically-changing nonuniform grids. Nonetheless, it is believed that unstructured adaptive-grid techniques will constitute a significant fraction of future high-pertormance supercomputing.

As an example, if a full-scale problem in computational fluid dynamics were to be solved efficiently in parallel, dynamic mesh adaptation would cause load imbalance among processors. This, in tum, would require large amounts of data movement at runime. It is therefore imperative to have an efficient dynamic load balancing mechanism as part of the solution procedure. However, since the computational mesh will be trequently adapted tor unsteady flows, the runtime load also has to be balanced at each step. In other words, the dynamic load balancing procedure itself must not pose a major overhead. This motivates our work.

We have developed a novel method, called PLUM [7], that dynamicially baliances processor workloads with a global view when pertonning adaptive numerical calculattions in il parallel messige-passing environment. Exanıining the pertormance of PLUM for an actual workload. which sinulates in dicoustic wind-tunnel experiment of a helicopter rotor blade. on threc different parallel machines dernonsirales that it cau be successfully ported without any code modifications.

We propose another new approach to dynamic load balancing lor unstructured grid applications based on defining a rohusl communicition pallem (lugicall or physical) :ameng prexessors, called symmetric broudcast nemorks $(S B N)$ |3. It is adaptive and decentralied in natture. and 
can be ported to any topological architecture through efficient embedding techniques. Portability results tor an adaptive unsteidy grid workload, generated by propagating a simulated shock wave through a tube, show that our approach reduces the redistribution cost at the expense of a minimal extra communication overhead. In many mesh adaptation applications in which the dara redistribution cost dominates the processing and communication cost, this is an acceptable trade-off.

\section{Architecture-Independent Load Balancer}

PLUM is an automatic and portable load balancing environment, specifically created to handle adaptive unstructured grid applications. It differs trom most other luad balancers in that it dynamically balances processor workloads with a global view $[1,7]$. In this paper, we examine its architecture-independent feature by comparing results for a rest case running on an SP2, Origin2000, and T3E.

PLUM consists of a partitioner and a remapper that load bal ance and redistribute the computational mesh when necessary. After an initial partitioning and mapping of the unstructured mesh, a solver executes several iterations of the application. A mesh adaptation procedure is invoked when the mesh is desired to be retined or coarsened. PLUM then gains control to determine if the workload among the processors has become unbal anced due to the mesh adaptation, and to take appropriate action. If load balancing is required, the adapted mesh is repartitioned and reassigned among the processors so that the cost of data movement is minimized. If the estimated remapping cost is lower than the expected computational gain to be achieved, the grid is remapped among the processors before solver execution is resumed.

Extensive details about PLUM are given in [7]. For completeness, we cnumerate some of its salient leatures.

- Reusing the Initial dual graph: PLUM repearedly utilizes the dual of the initial mesh for the purposes of load balancing. This keeps the complexity of the partitioning and reassignment phases constant during the course of an adaptive computation. New computational grids obtained hy adaptation are translated by changing the weights of the vertices and edges of the dual graph.

- Parallel mesh repartitioning: PLUM can use any general-purpose partitioner that balances the computationd loads and minimizes the nutime interprocessor communication. Several excellent parallel partitioners are now avisiahle $[4,5,101$; however, the restils presented in this paper use PMeTiS $[6]$.

- Processor remapping and data movement: To map new partitions to processors while mininu ang the cost of redistrihution. PLUM tirst constnucts a similarity matrix. This maltix indicalles how the vertex weights of the new subdentains are distrihuted over lle processors. There general metrics: TotalV, MaxV, and MaxSR, are used to model the remapping cost on most multiprocessor systems [8]. Both optimal and heuristic al gorithms for minimizing these metrics are available within PLUM.

- Cost model metrics: PLUM pertorns data remapping in a bulk synchronous fashion to amortize message start-up costs and obtain good cache pertormance. The procedure is similar to the superstep model of BSP [9]. The expected redistribution cost tor a given architecture can be expressed as a linear tunction of MaxSR. The machinedependent parameters are determined empirically.

\subsection{Helicopter Rotor Test Case}

The computational mesh used to evaluate the PLUM load balancer is the one used to simulate an acoustics windtunnel experiment of a UH- $1 \mathrm{H}$ helicopter rotor blade [7]. A cut-out view of the initial tetrahedral mesh is shown in Fig. 1. Three refinement strategies, called Real_1, Real_2. and Real_3, are studied. each subdividing varying fractions of the domain based on an error indicator calculated from the flow solution. This increased the number of mesh elements from 60,968 to $82,489,201,780$, and 321,841 , $\pi$ spectively.

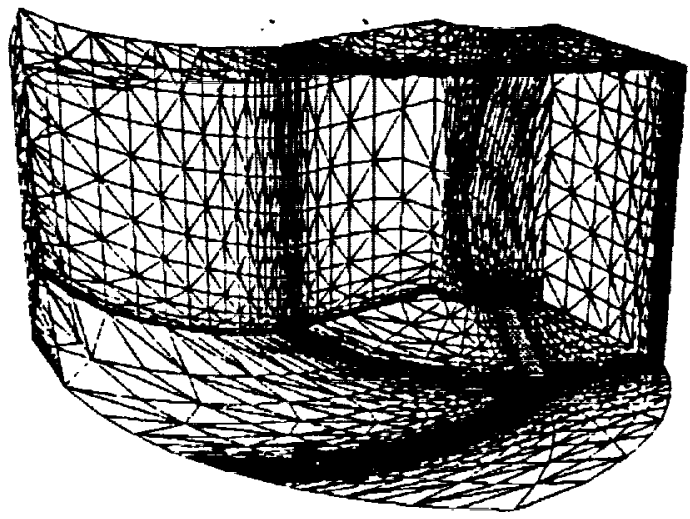

Figure 1. Cut-out view of the initial mesh for the helicopter rotor blade experiment.

\subsection{Experimental Results}

The three left plors in Fig. 2 illustrate parallel speedup for the three edge-marking strategies on an SP2. Origin2000, and T3E. Two sets of results are presented for each machine: one when datti remapping is pertiomled atter mesh retinement, and the other when remapping is done betore refincrnent. The speedup numbers are atmost identical on all three makhines. The Real_3 calse shows the hest specdup values teviluse it is the mest computation inconsive. Remap-

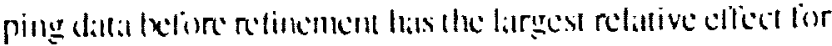


Real_1, because it has the smallest refinement region and it returns the biggest benefit by predictively load balancing the refined mesh. The best results are for Real_3 with remapping before refinement, showing an efficiency greater than $87 \%$ on 32 processors.
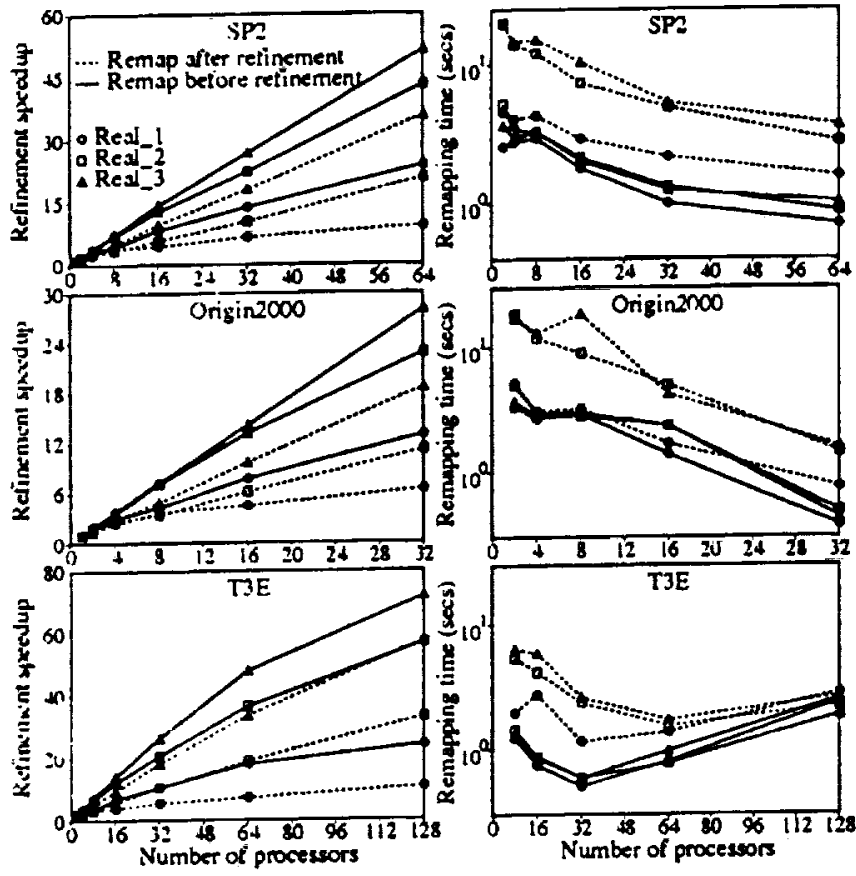

Figure 2. Refinement speedup (left) and remapping time (right) within PLUM on an SP2, Origin2000, and T3E, when data is redistributed after or before mesh refinement.

The three right plots in Fig. 2 show the corresponding rem:ipping times. In almost every case, a significant reduction in remapping time is observed when the adapted mesh is load balanced by performing data movement prior tn refinement. This is because the mesh grows in size only atter the data has been redistributed. In general, the remapping times also decrease as the number of processors is increased. This is because event though the total volume of data movement increases with the number of processors, there are actually more processors to share the work.

Perhaps the most remarkable feature of these results is the peculiar telalvior of the T3E when $P \geq 64$. When usIny up to 32 nrocessors, the remapping pertormance of the $T .3 E$ is ver sirrilar to that of the other two machines. However. tor $l^{\prime}=6.1$ and 128 , the remapping overhead begins (1) increalse and violates our cost model. The runtime diflierence when diata is remapped hetore and after retinement is drarnatically diminished; in bact, all the remapping times Ix gin fo converge to a single valluc! This indicattes that the remippine tinc is also aflected by the interprocessor communication pillem. One solution would the to take aldvals- rage of the T3E's ability to etticiently pertionn one-sided communicalion.

\section{Topology-Independent Load Balancer}

In this section, we describe a dynamic load balancer based on a symmetric broalcast network (SBN), which takes into account the global view of system loads among the processors. The SBN is a robust, topology-independent communication partern (logical or physical) among $P$ processors in a multicomputer system [3]. An SBN of dimension $d \geq 0$, denoted as $\operatorname{SBN}(d)$, is a $(d+1)$-stage interconnection network with $P=2^{d}$ processors in each stage. It is constructed recursively as follows. A single node forms the basis network $\operatorname{SBN}(0)$. For $d>0$, an $\operatorname{SBN}(d)$ is obtained trom a pair of $\operatorname{SBN}(d-1) s$ by adding a communication stage in the front with the following additional interprocessor connections: (i) node $i$ in stage 0 , is made adjacent to node $j=(i+P / 2) \bmod P$ of stage 1 and (ii) node $j$ in stage 1 is made adjacent to the node in stage 2 which was the stage 0 successor of node $i$ in $\operatorname{SBN}(d-1)$.

The proposed SBN-based load balancer takes into accoumt a global view of the system and makes it effective for adaptive grid applications. Prior work [2] has demonstrated the viability of this SBN approach. It processes two types of messages: (i) load balance messages when a load imbalance is detected, and (ii) job distribution messages to reallocate jobs. We give a brief description of the various parameters and policies involved in our implementation.

- Weighted queue length: This is to take into account al! the system variables like computation, communication, and redistribution costs, that affect the processing of a local queue. Note that no redistribution cost is incurred if the data set is available on the local processor. Similarly, the communication cost is zero if the data sets of all adjacent vertices are stored locally.

- Prioritized vertex selection: When selecting vertices to be processed, the SBN load balancer takes advantage of the underlying structure of the adaptive grid and deters local execution of boundary vertices as long as possible becalluse they may be migrated for more efficient execution. Thus, vertices with no communication and redistribution costs are executed first.

- Differential edge cut: This is the total change in the communication and redistribution costs if a vertex were moved trom one processor to another. The vertex with the smallest differential edge cut is chosen tor migration. This policy strives to maintain or improve the cur size during the excecurion of the load balducing al gorithm.

- Data redistribution policy: Dilla redistrihution is perfonted in al lase mammer, i.c., the nort-locial datal seet loor at vertex is not moved to a processor until it is abmot to the exectuced. Furthemore, the datla sets of all idfiatem ver- 
tices are also migrated at that time. This policy greatly reduces the redistribution and communication costs by avoiding multiple migrations of data sets.

\subsection{Unsteady Simulation Test Case}

To evaluate the SBN tramework, a computational grid is used to simulate an unsteady environment where the adapted region is strongly time-dependent. This experiment is performed by propagating a simulated shock wave through the initial grid shown at the top of Fig. 3. The test case is generated by refining all elements within a cylindrical volume moving left to right across the domain with constant velocity, while coarsening previously-refined elements in its wake. Performance is measured at nine successive adaptation levels during which the mesh increases from 50,000 to $1,833,730$ elements.

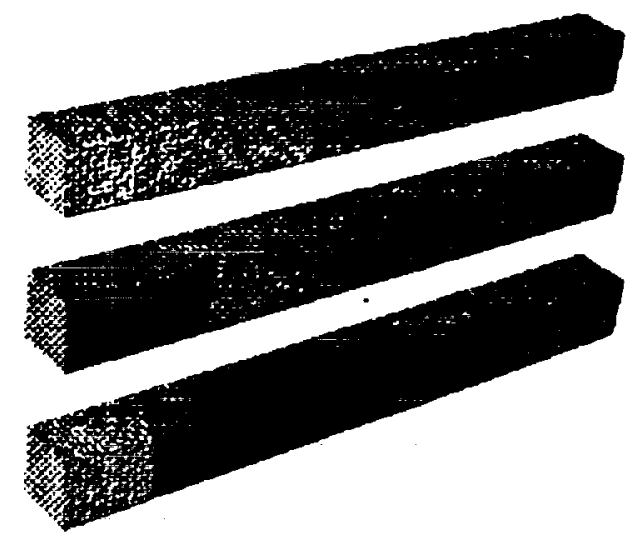

Figure 3. Initial and adapted meshes (after levels 1 and 5) for the simulated unsteady experiment.

\subsection{Experimental Results}

Table 1 presents performance results on an SP2, averaged over the nine levels of adaptation. In addition so achieving excellent load balance, the redistribution cost (expressed as MaxSR, the maximum number of vertices moved in and out of any processor) is significantly reduced. However, the edge cut percentages are somewhat higher, indicating that the SBN strategy reduces the redistribution cost at the expense of a slighily higher communication cost.

Table 2 gives the number of bytes that were transferred between processors during the load balancing and the job distribution phases. The number of bytes translemed is also expressed ats a percentage of the available bandwidtl. (A wide-node SP2 hals a messige bandwidth of 36 megahyle:s scoond and a message latency of 4 ) micer

\begin{tabular}{|c|c|c|c|c|}
\hline \multirow[b]{2}{*}{$\mathbf{P}$} & \multicolumn{2}{|c|}{ Edge Cut } & \multirow[b]{2}{*}{ MaxSR } & \multirow{2}{*}{$\begin{array}{c}\text { Load } \\
\text { Imbalance }\end{array}$} \\
\hline & Betore & After & & \\
\hline 2 & $2.88 \%$ & $\overline{5.51 \%}$ & 80,037 & 1.00 \\
\hline 4 & $7.27 \%$ & $10.76 \%$ & 76,665 & 1.00 \\
\hline 8 & $12.71 \%$ & $16.35 \%$ & 53,745 & 1.00 \\
\hline 16 & $19.40 \%$ & $23.87 \%$ & 46,825 & 1.01 \\
\hline 32 & $24.42 \%$ & $30.41 \%$ & 28.031 & 1.02 \\
\hline
\end{tabular}

Table 1. Grid adaptation results on an SP2 using the SBN-based load balancer.

seconds.) The results demonstrate that the cost of vertex migration is significantly greater than the cost of actually balancing the system load. An extrapolation of the results using exponential curve-fitting indicates that normal speedup will not scale for $P>128$.

\begin{tabular}{||r|c|c|c|r||}
\hline \hline & \multicolumn{2}{|c|}{ Balancing Messages } & \multicolumn{2}{c||}{ Migration Messages } \\
\cline { 2 - 5 } $\mathrm{V}$ & Volume & Bandwidth & Volume & Bandwidth \\
\hline \hline 2 & $0.342 \mathrm{MB}$ & $0.00 \%$ & $3.919 \mathrm{MB}$ & $3.67 \%$ \\
4 & $0.150 \mathrm{MB}$ & $0.00 \%$ & $7.939 \mathrm{MB}$ & $7.44 \%$ \\
8 & $0.463 \mathrm{MB}$ & $0.01 \%$ & $25.397 \mathrm{MB}$ & $23.79 \%$ \\
16 & $0.581 \mathrm{MB}$ & $0.02 \%$ & $30.454 \mathrm{MB}$ & $28.53 \%$ \\
32 & $1.550 \mathrm{MB}$ & $0.12 \%$ & $38.244 \mathrm{MB}$ & $35.83 \%$ \\
\hline
\end{tabular}

Table 2. Communication overhead of the SBN load balancer.

Table 3 shows the percentage of time spent in the SBN load balancer compared to the execution time required to process the mesh adaptation application. The three columns correspond to three types of load balancing activities: (i) the time needed to handle balance related messages, (ii) the time needed to migrate messages from one processor to another, and (iii) the time needed to select the next vertex to be processed. The results show that processing related to the selection of vertices is the most expensive phase of the SBN load balancer. However, the total time required to load balance is still relatively small compared to the time spent processing the mesh.

We were able to directly port the SBN methodology from an SP2 to an Origin2000 without any code modifications. This demonstrates the architecure independence of our load balancer. The load imbalance factors were almost identical on the two machines. Tle edge cut vad ues were consistently lirger on the Origin2 $(x)$ ), but the MaxSR villues were lirger only when using less thin 16 processors.

Some of the diflerences in pertiontance results on these 


\begin{tabular}{||r|c|c|c||}
\hline \hline $\mathrm{P}$ & $\begin{array}{c}\text { Balancing } \\
\text { Activity }\end{array}$ & $\begin{array}{c}\text { Migration } \\
\text { Activity }\end{array}$ & $\begin{array}{c}\text { Vertex } \\
\text { Selection }\end{array}$ \\
\hline \hline 2 & $0.0053 \%$ & $0.0014 \%$ & $0.4530 \%$ \\
4 & $0.0087 \%$ & $0.0069 \%$ & $2.0381 \%$ \\
8 & $0.1745 \%$ & $0.0569 \%$ & $2.8386 \%$ \\
16 & $0.2669 \%$ & $0.0629 \%$ & $0.8845 \%$ \\
32 & $0.1154 \%$ & $0.0774 \%$ & $2.1043 \%$ \\
\hline \hline
\end{tabular}

Table 3. Overhead of the SBN load balancer.

two machines are due to additional refinements that were implemented prior to running the experiments on the Origin2000. First, the algorithm for distributing jobs was modified to guarantee that the processor initiating load balancing always had sufficient workload. This reduces the total number of balancing related messages but increases the number of vertices migrated, especially for small numbers of processors. Second, data sets corresponding to groups of vertices were moved at a time. This bulk mi gration strategy reduces the volume of migration messages by more than $\$ 0 \%$ in otir experiments.

\section{PLUM vs. SBN}

Let us highlight some of the differences between the two dynamic load balancers described in this paper.

- Processing is tenipurarily halted under PLUM while the load is being balanced. The SBN approach. on the other hand, allows processing to continue asynchronously. This feature allows for the possibility of utilizing latencytolerant techniques to hide the communication overhead of redistribution.

- Under PLUM, suspension of processing and subsequent repartitioning does not guarantee an improvement in the quality of load balance. In contrast, the SBN approach always results in improved load balance.

- PLUM redistributes all necessary data to the appropriate processors immediately before processing. SBN, however, migrates data to a processor only when it is ready to process $i t$, thus reducing the redistribution and communication overhead.

- PLUM, unlike SBN, periorms remapping predictively. This results in a significant reduction of data movement during redistribution and improves the overall efficiency of the refinement procedure.

- Load balincing under PLUM occurs before the solver phlase of the computation, whereas SBN balinces the load during the solverexecution. We theretore cannot directly compare PLUM and SBN-hased load halancing, sinke their relative pertionnanec is solver dependent.

\section{Summary}

We have demonstrated the portability of our novel approaches to solve the load balancing problem for adaptive unstructured grids on three state-of-the-art commercial parallel machines. The experiments were conducted using actual workloads of both steady and unsteady grids obtained from real-life applications. We are currently examining the portability of our software on other platforms (such as shared-memory environments or networks of workstations) as well as employing various parallel programming models.

\section{Acknowledgements}

This work is suppored by NASA under Contract Numbers NAS 2-14303 with MRJ Technology Solutions and NAS 2-96027 with Universities Space Research Association, and by Texas Advanced Research Program Grant Number TARP-97-003594-013.

\section{References}

[1] R. Biswas and L. Oliker. Experiments with repartitioning and load balancing adaptive meshes. Technical Report NAS. 97-021, NASA Amès Reseaich Center. 1997.

[2] S. Das, D. Harvey, and R. Biswas. Parallel processing of adaptive meshes with load balancing. In 27th intl. Conf. on Parallel Processing, pages 502-509. 1998.

[3] S. Das and S. Prasad. Implementing task ready queues in a multiprocessing environment. In Intl. Conf. on Parallel Computing, pages 132-140. 1990.

[4] J. Flaherty, R. Loy, C. Ozturan, M. Shephard, B. Szymanski. J. Teresco, and L. Ziantz. Parallel structures and dynamic load balancing for adaptive finite element computation. Applied Numerical Marhematics, 26:241-263, 1998.

[5] G. Karypis and V. Kumar. A fast and high quality multilevel scheme for partitioning irregular graphs. Technical Report 95-035. University of Minnesota, 1995.

[6] G. Karypis and V. Kumar. Parallel multilevel k-way partitioning scheme for irregular graphs. Technical Report 96036. University of Minnesota. 1996.

[7] L. Oliker and R. Biswas. PLUM: Parallel load balancing for adaptive unstiuctured meshes. Journal of Parallel and Distributed Compuring. 52:150-177, 1998.

[8] L. Oliker, R. Biswas, and H. Gabow. Performance analysis and portability of the PLUM load balancing system. In Euro-Par'98 Parallel Processing. Springer-Verlag LNCS 1470:307-317. 1998.

[9] [.. Valiant. A bridging model for parallel computation. Com. munications of the ACM. 33:103-111. 1990.

flol C. Wialshaw, M. Cross, and M. Everelt. Parallel dynamic graph pattitioning for adaptive unst ruclured nesles. Jeurnet of ferrulld and Distribuscd Compuring. 47:102-108. [(x)7. 
